\title{
Inflammatory Proteins: Chemical and Biological Aspects*
}

\author{
PETER A. WARD
}

Department of Pathology, The University of Michigan Medical School, Ann Arbor, Michigan 48109

\begin{abstract}
Mediators of the inflammatory system can be broadly described as those that pre-exist and are released by active secretory mechanisms from storage granules (as in the case of vasoactive amines within cytoplasmic granules of mast cell), those that are biosynthetically generated de novo (as in the case of prostaglandins) and those that are produced by enzymatic cleavage of a precursor protein. With regard to the last category, cleavage of the precursor protein releasing a biologically active fragment occurs following activation of a plasma proenzyme or the release of a stored protease from within a cell.
\end{abstract}

\section{Types OF Inflammatory Mediators}

IN ADDITION TO DIFFERENCES IN THE MANNER OF GENERATION, inflammatory mediators also can be grouped into at least three different categories, based upon their biological effects. They demonstrate (1) a vasopermeability activity, or (2) they are chemotactically active, or (3) their function is to modulate the effects of other inflammatory mediators on effector cells (reviewed, 1). The last category is well demonstrated by the ability of prostaglandins to cause an augmentation in the contractile response of smooth muscle to kinins, and by the ability of prostaglandins (e.g. PG $E_{1}$ ) tc diminish the response of a neutrophil to a chemotactic stimulus(2). Thus, the inflammatory system of mediators is complex with many intersecting pathways, much like a network of electrical circuits in which a single defect is not necessarily translated into a proximate outcome. Single point abnormalities in the inflammatory system are often recognized clinically only as obscure expressions of the underlying defect.

\section{Chemotactic Mediators}

Over the past decade, our understanding of the inflammatory system has been greatly facilitated by knowledge of the chemotactic system and its vital role in the inflammatory process. Chemotactic mediators derive from multiple sources, including complement proteins, bacteria and lymphoid cells (reviewed, 3). In the case of bacteria, peptides of low molecular weight are elaborated from a variety of bacteria as synthetic products and have a potent influence in attracting leukocytes to locales containing bacteria. Lymphoid cells stimulated by contact with antigen produce chemotactic factors for a variety of leukocytes, the most active being chemotactic factors for monocytes and for $T$ lymphocytes. Protein synthesis is a requirement for the production of these lymphoid cell-derived factors. Although their molecular weights vary from around 20,000

*Supported in part by NIH Grant AI-09651 to 150,000 , their structures have been not defined. Most of our information regarding naturally-occurring chemotactic factors has come from detailed studies indicating that activation of the classical complement pathway (by immune complexes) and the alternate complement pathway (by lipids, polysaccharides and lipopolysaccharides) results in the formation of a peptide (C5a) that has potent chemotactic activity (4). It is also clear that this product is a derivative of the fifth (C5) component of complement. In the case of the C5 generated chemotactic peptide there is no question that this peptide derives from proteolysis of the C5 molecule. The precise nature of C5 cleaving enzyme generated from either of the pathways of complement activation is not known, since in the case of the classical pathway the C5 cleaving enzyme contains modified forms of the second (C2), the third (C3) and the fourth (C4) components of complement while in the case of the alternate pathway a form of C3 (C3b) and a fragmentation product of another factor $(B)$ with or without yet a third protein (properdin) constitute the C5-cleaving enzyme. While the evidence is not definitive, these two enzyme-like entities appear to cause proteolysis of C5 at a single point in the a chain of $\mathrm{C5}$, resulting in a small (C5a) and a large (C5b) fragment. This proteolysis is not associated with a trypsin-like enzyme, since other trypsin-susceptible substrates are not cleaved, and trypsin inhibitors and serine esterase inhibitors do not block the cleavage of $\mathrm{C5}$. A third species of C5 cleaving activity is the neutral proteases contained within lysosomal granules of neutrophils. In human leukocytes, the major neutral protease of lysosomal granules, which has been identified as elastrase, will also cleave $\mathrm{C} 5$ producting a chemotactic peptide (5). The biologically active fragment is probably closely related structurally to the C5a peptide but is almost surely not structurally identical with C5a. Thus, it appears that there exists a family of chemotactic peptides, all presumably with a common core from the $\mathrm{N}$-terminal region of the $\alpha$ chain of C5 (6).

What is the chemical nature of C5a? Encompassing only $5 \%\left(M_{r} 12,500\right)$ of the total mass of the C5 molecule, C5a is an extremely cationic peptide whose amino acid composition and sequence have been described (7). The $\mathrm{N}$-terminal portion of $\mathrm{C} 5 \mathrm{a}$ is structurally identical with the original region of the $\alpha$ chain of the C5 molecule and contains a disulfide loop with 5 half cystines near this region. Little is known regarding the role and the requirement for this portion of the molecule in the biological activity associated with C5a. However, the C-terminal region of the C5a peptide is critical, since deletion of the $\mathrm{C}$-terminal arginine reduces the chemo- 
tactic activity of C5a by a factor of 40 fold. Any further cleavage of the remaining $\mathrm{C}$-terminal residues abolishes altogether the residual chemotactic activity. Synthetic reconstruction of the C-terminal pentapeptide of C5a does not provide a product with biological activity, indicating that additional portions of C5a are essential for its biological activity.

Activation of the complement system in serum or plasma results in generation of the chemotactic peptide, C5a, but this peptide undergoes metabolic conversion to C5a des arg (8). In other words, the C-terminal arginine of $\mathrm{C} 5 \mathrm{a}$ is removed by the action of a plasma carboxypeptidase $\mathrm{N}$-type activity, which was originally described as a kininase activity due to its ability to inactivate bradykinin by the same modification (removal of the carboxyl-terminal, arginine). C5a des arg is 40 fold less active chemotactically when compared to C5a. However, it has recently been suggested that C5a des arg immediately interacts with an acidic protein to form a complex, which regains full chemotactic activity relative to $\mathrm{C5a}$ (9). If this is indeed the case, it is unclear what biological advantage results from the metabolic conversion of C5a, except possibly for the fact that the second biological function of C5a, namely its anaphylatoxin activity (defined by the ability to contract smooth muscle and to cause of histamine from mast cells) is largely destroyed by conversion of C5a to C5a des arg. The anaphylatoxin activity does not reappear following the putative formation of the complex between C5a des arg and the acidic protein.

\section{Regulation of the C5a Chemotactic Peptide}

The metabolic convorsion of C5a, as described above, results in a product that is ultimately preserved as a chemotactic agent but rendered largely inactive in terms of its vasopermeability (anaphylatoxin) activity. However, because C5a (or its conversion product) is extraordinarily biologically active as a chemotactic peptide (in the range of $10^{-11} \mathrm{M}$ ), it can be assumed that rigorous regulatory control of this peptide exists in order to prevent uncontrolled triggering of the inflammatory system. Indeed, there is evidence that serum factor, the chemotactic factor inactivator (CFI), regulates the level of $\mathrm{C} 5 \mathrm{a}$ and related chemotactic peptides in serum (10). CFI is a large protein with an estimated molecular weight of 140,000 daltons and it interacts with C5a or with C5a des arg to irreversibly inhibit the chemotactic activity. Because homogenous preparations of CFI almost invariably contain traces of carboxypeptidase, it is not known if CFI acts directly with C5a or with C5a des arg. The end result is an alteration of the peptide that precludes expression of chemotactic activity.

CFI exists as a trace protein in serum. A volume of $0.1 \mathrm{ml}$ serum will inactivate $3 \mathrm{ED}_{50}$ 's of a C5a preparation, which can be calculated to contain approximately $150 \mathrm{ng}$ C5a. This can be roughly calculated to represent a ratio of C5a to CFI of $100: 1$. At $37^{\circ} \mathrm{C}$, inactivation of C5a by CFI occurs within minutes of mixing. In view of these data, why it is indeed possible to generate by complement activation any stable chemotactic activity in whole serum is puzzling (11). However, there is recent evidence that products of CFI-induced inactivation of
CFI interfere with further CFI activity, but no definitive evidence exists. In spite of this apparent discrepancy, patients who have excessive (above the normal, low) levels of CFI in their serum often demonstrate marked defects in their ability to develop acute inflammatory responses, suggesting that CFI does indeed play an important regulatory role in the inflammatory system. For instance, patients with lepromatous leprosy contain high serum levels of CFI. When compared with normal controls or with patients with tuberculoid leprosy (these patients do not have elevated serum levels of CFI), mobilization of neutrophils in acute inflammatory reactions in the skin results in approximately $50 \%$ fewer neutrophils in patients with lepromatous leprosy as compared with the two other groups in which elevations in CFI levels are not present (12). Additional evidence favoring CFI as an important regulator of the inflammatory response comes from studies of experimental animals in which CFI is able to block completely the acute inflammatory response to deposition of immune complexes in dermal vessels and in pulmonary alveoli (13).

\section{Interactions of Chemotactic Factors with Neutrophils}

The interactions of C5a with the neutrophil has been studied extensively over the past few years (reviewed, 14). There is clear evidence that receptors for C5a exist on the surface of the human neutrophil, numbering between 20,000 and 100,000 receptors per cell. Very similar results with the synthetic chemotactic factor peptide, $\mathrm{N}$-formyl-methionyl-leucyl-phenylalanine, have been found. It is quite clear that the receptors for these two chemotactic peptides are not identical. The precise delineation of receptor binding data has been difficult, because at $37^{\circ} \mathrm{C}$ bound $\mathrm{C5a}$ (as well as the synthetic chemotactic peptide) is internalized rapidly and appears to undergo hydrolysis in the process. Nevertheless, the occupancy of the C5a receptor on the cell is associated with cationic fluxes across the cell membrane, with $\mathrm{Ca}^{++}$flux being perhaps the key event.

As flux occurs across the external cell membrane, it is also occurring within the cell across the intracytoplasmic membranes, and it seems likely that calmodulin is involved in this process. As has been well documented in skeletal muscle, the ultimate outcome of these events is a contractile response of the cell. In the neutrophil this appears to be related to the formation of condensed actin filaments within the subcortical region of the cell, presumably in proximity to the surface of the cell where the receptors have been occupied by the chemotactic peptide.

Other events also occur within neutrophil. For instance, the cell is stimulated (for reasons that are not understood) to secrete lysosomal enzymes to the cell's exterior. However, the amount of enzyme release is relatively limited unless the cell is first treated with cytochalasin B. Another series of responses of the cell to the chemotactic peptide is abrupt increase in cell respiration. $\mathrm{O}_{2}$ uptake increases dramatically, together with production of $\mathrm{CO}_{2}$, and the pentose shunt becomes activated. Perhaps the most important outcome of these events is the production of oxygen free radicals, in- 
cluding such products as $\mathrm{O}_{\overline{2}}, \mathrm{OH} \bullet, \mathrm{O}_{2}$ as well as several other short-lived materials. From this biochemical repertoire it is possible to quantitate the responses by measuring chemiluminescence, which is possible by liquid scintillation methodologies. The biological significance of production highly reactive oxygen products is probably related to the fact that these materials are extremely toxic to tissues and may be directly contributory to the production of tissue injury by lipid peroxidation of cell membranes and other vital tissue constituents. It is fair to say that the roles of the oxygen metabolites in the inflammatory process are only dimly understood at present.

\section{Abnormalities of the Inflammatory System}

With the complicated array of events described above, it is of little surprise that any number of abnormalities of the inflammatory response have been recognized. These can be broadly classified as: (1) deficiency of substrate needed for mediator production; (2) abnormality of the substrate; and (3) the presence of regulatory defects involving plasma proteins or effector cells (leukocytes) (reviewed, 14). With regard to the first type of defect, a $\mathrm{C} 4$ deficiency syndrome has been described in both mice and men. In mice, this deficiency state is associated, as might be expected, with the inability of mice to develop acute, neutrophil-rich, inflammatory reactions in response to the presence of immune complexes in tissues (15). In humans the absence of $\mathrm{C5}$ is related to susceptibility to bacterial infections with Nisseria $s p$., but the precise explanation for this observation is not known. Although these represent the only documented examples of genetically determined deficiency states of the C5 substrate, many examples of deficiencies involving other substrates for the inflammatory system are known, many involving diminished levels of $\mathrm{C} 3$ for example. The general impression remains that animals and humans with the absolute deficiency states are remarkably healthy, probably a manifestation of the interlocking nature of the mediator pathways such that a single defect in the system is not directly expressed in vivo in contrast to the ready demonstration of the defect in vitro. A companion to defects involving substrate deficiencies is the presence of abnormal substrates. In the case of C5, some limited information is available. Several years ago a series of children were described with problems of skin infections that were linked to the presence of an abnormal C5. This was identified by the reduced ability to support in vitro the opsonization and subsequent phagocytic uptake of yeast cells into leukocytes when yeast particles were first treated with serum containing the putatively abnormal C5 (16). These results were in marked contrast to the results obtained when reference normal control sera were used. Although the precise nature of the C5 defect has not been described (indeed there has been considerable controversy over these studies), this example illustrates that abnormalities in protein substrates represent, at least theoretically, one type of aberration in the inflammatory system.

The third general mechanism of defects in the inflammatory system involves regulatory defects. These have been alluded to above with examples of patients with abnomally high levels of the chemotactic factor inactivator (CFI). These individuals behave in a manner indicating that they cannot effectively mobilize inflammatory cells into locales of tissue damage or into sites containing bacteria. These patients manifest cutaneous anergy. Indeed, as described above, the numbers of neutrophils accumulating in acutely inflamed skin sites are appreciably less than half the number found in patients with normal serum levels of CFI. The clinical experience, which is quite limited, suggests that the defect is a quantitative one. Presumably sufficient numbers of neutrophils can usually be mobilized into locales to provide protection against bacterial invaders, but, given the appropriate circumstances, the number of leukocytes mobilized is insufficient to produce the expected intensity of the inflammatory response.

The last broad category of abnormalities in the inflammatory system involves defects in the effector cells, the leukocytes. There are many types of defects, most being of an acquired nature, but some include genetically determined abnormalities. These defects render the leukocytes relatively nonresponsive to chemotactic stimuli. As a result, there may be serious impairments in host defenses because of the inability to mobilize adequate numbers of inflammatory cells. This is most tellingly demonstrated in acute granulocyte leukemia when the immature granulocytes are devoid of chemotactic responsiveness (17). The outcome is, as expected, the development of fatal bacterial infections. Acquired chemotactic defects in leukocytes have been associated with metabolic disorders (such as diabetes mellitus and uremia), the shock syndrome, acute or chronic malnutrition, acute viral infections (especially influenza), malignant tumors, and many other conditions. The mechanisms underlying these acquired defects are quite clearly diverse in nature.

Genetically determined chemotactic defects include the Chediak-Higashi syndrome and the actin-dysfunction syndrome. How the former can be explained is not altogether clear unless one accepts that the underlying problem is with defective microtubules, since there is evidence that intact microtubules are somehow tied to the chemotactic responsiveness by a leukocyte.

The cleavage of a plasma protein substrate resulting in the release of a peptide such as $\mathrm{C} 5 \mathrm{a}$ has dramatic consequences, as outlined above. Unraveling the details from beginning to end is starting to give us totally new perspectives of the inflammatory system. This knowledge will probably ultimately revolutionize our approach to therapeutic manipulation of the inflammatory system. However, this will not be possible until we have many more facts in hand.

\section{REFERENCES}

1. Ward, P.A. "Inflammation", In Principles of Pathobiology, M.F. LaVia and R.B. Hill (editors), Oxford Press, Third edition, pg. 112-199 (1980).

2. Kunkel, S.L., Thrall, R.S., Kunkel, K.G., MeCormick, J.R., Ward, P.A., and Zurier, R.B. Suppression of immune complex vasculitis in rats by prostaglandin. J. Clin. Invest. 64, 1525-1529 (1979).

3. Ward, P.A., and Becker, E.L. Biology of leukotaxis. Rev. Physiology, Biochemistry and Pharmacology 77, 125-148 (1977). 
4. Hugli, T.E., and Muller-Eberhard, H.J. Anaphylatoxins: C3a and C5a. Advances in Immunology 26, 1-53 (1978).

5. Brozna, J.P., Senior, R.M., Kreutzer, D.L., and Ward, P.A. Chemotactic factor inactivator of human granulocytes. $J$. Clin. Invest. 60, 1280-1288 (1977).

6. Phan, S.H., and Ward, P.A. Generation of biological activity from the purified a chain of C5. J. Immunol 123, 2735-2740 (1979).

7. Fernandez, H., and Hugli, T.E. Partial characterization of human C5a anaphylatoxin. I. Chemical description of the carbohydrate and polypeptide portions of human $\mathrm{C} 5 \mathrm{a} J$. Immun. 117, 1688-1694 (1976).

8. Fernandez, H.N., and Hugli, T.E. Primary structural analysis of the polypeptide portion of human C5a anaphylatoxin. J. Biol Chem. 253, 6955-6964 (1978).

9. Perez, H.D., Goldstein, I.M., Chernoff, D., Webster, R.O., and Henson, P.M. Chemotactic activity of C5a des arg: Evidence of a requirement for an anionic peptide "helper factor" and inhibition by a cationic in serum from patients with systemic lupus erythematosus. Molec. Immunol (In press).

10. Berenberg, J.L., and Ward, P.A. 'I'he chemotactic factor inactivator in normal human serum. $J$. Clin. Invest. 52, 1200-1206 (1973).

11. Kreutzer, D.L., Claypool, W.D., Jones, M., and Ward, P.A.
Isolation by hydrophobic chromatography of the chemotactic factor inactivators from human serum. Clin. Immunol Immunopath. 12, 162-176 (1979).

12. Bullock, W.E., Ho, M.F., and Chen, M.J. Studies of immune mechanisms in leprosy. Quantitative and qualitative studies of the local cellular exudative response in leprosy. J. Reticulo-endothelial. Soc. 16, 259-265 (1974).

13. Johnson, K.J., Anderson, T.P., and Ward P.A. Suppression of immune complex-induced inflammation by the chemotactic factor inactivator. J. Clin. Invest. 59, 951-958 (1977).

17. Becker, E.L., and Ward, P.A. "Complement and chemotaxis", In Clinical Immunology and Immunopathology, C. Parker (editor), W.B. Saunders Co., 1, 272-297 (1980).

15. Larsen, G.L., Mitchell, B.C., and Henson, P.M. The pulmonary response of $\mathrm{C} 5$ sufficient and deficient mice to immune complexes. Fed. Proceed. 39, 333 (abstract) (1980).

16. Miller, M.E., Seals, J., Kaye, R., and Levitsky, L.C. A familial plasma-associated defect of phagocytosis. Lancet 2, 60 (1968).

17. Ward, P.A. Leukotactic factors in health and disease, Presented at the Parke-Davis Award Lecture (American Society for Experimental Pathology), Chicago, April, 1971. Amer. J. Pathol. 64, 521-530 (1971). 